\title{
Study of Charmed Baryons at Belle
}

\author{
Mizuki Sumihama, for the Belle collaboration* \\ Gifu university and RCNP Osaka university \\ E-mail: sumihama@rcnp.osaka-u.ac.jp
}

We report recent results on charmed baryons based on the data sample collected with the Belle detector at the KEKB asymmetric energy $e^{+} e^{-}$collider. These include the search for a hidden strange pentaquark $P_{s}^{+}$via $\Lambda_{c}^{+} \rightarrow \phi p \pi^{0}$ decay, the measurement of the branching fractions of hadronic decays of the $\Omega_{c}^{0}$ ground state, the observation of four states of $\Omega_{c}^{* 0}$ and $\Xi_{c}(2930)^{0}$, and the measurement of the production cross sections of hyperons and charmed baryons.

XXVI International Workshop on Deep-Inelastic Scattering and Related Subjects (DIS2018)

16-20 April 2018

Kobe, Japan

${ }^{*}$ Speaker. 


\section{Introduction}

The charmed baryon is a unique system, which may be interpreted as a heavy charm quark interacting with a light diquark due to the suppression of color spin interaction for the charm quark. The study of charmed baryons leads to the understanding of diquark picture of baryons. The hadronic decays of charmed baryons governed by the $\mathrm{c} \rightarrow \mathrm{s}$ quark transition are a good laboratory to study strange baryons. In this proceedings, the review of results for charmed baryons obtained by Belle collaboration are presented. The data is recorded with the Belle detector located at the KEKB asymmetric-energy $e^{+} e^{-}$collider.

\section{Search for strange pentaquark via $\Lambda_{c}^{+} \rightarrow \phi p \pi^{0}$ decay}

The discovery of two hidden-charm pentaquarks $P_{c}^{+}(4380)$ and $P_{c}^{+}(4450)$ by the LHCb collaboration [1] raises the question of whether a hidden-strangeness $(s \bar{s})$ pentaquark $P_{s}^{+}$exists. The decay of $\Lambda_{c}^{+} \rightarrow \phi p \pi^{0}$ is a candidate which can detect the $P_{s}^{+}$. We study the Cabibbo-suppressed decay $\Lambda_{c}^{+} \rightarrow \phi p \pi^{0}$ and the nonresonant $\Lambda_{c}^{+} \rightarrow K^{+} K^{-} p \pi^{0}$ decay [2]. No significant signal is observed, and we set an upper limit on the branching fraction of $\mathcal{B}\left(\Lambda_{c}^{+} \rightarrow \phi p \pi^{0}\right)<15.3 \times 10^{-5}$, and $\mathcal{B}\left(\Lambda_{c}^{+} \rightarrow K^{+} K^{-} p \pi^{0}\right)<6.3 \times 10^{-5}$ at $90 \%$ confidence level. We see no evidence for a hiddenstrangeness pentaquark decay of $P_{s}^{+} \rightarrow \phi p$ and set an upper limit on the product branching fraction of $\mathcal{B}\left(\Lambda_{c}^{+} \rightarrow P_{s}^{+} \pi^{0}\right) \times \mathcal{B}\left(P_{s}^{+} \rightarrow \phi p\right)<8.3 \times 10^{-5}$ at $90 \%$ confidence level. This limit is a factor of 6 higher than the product branching fraction measured by the $\mathrm{LHCb}$ for the $P_{c}^{+}(4450)$ state.

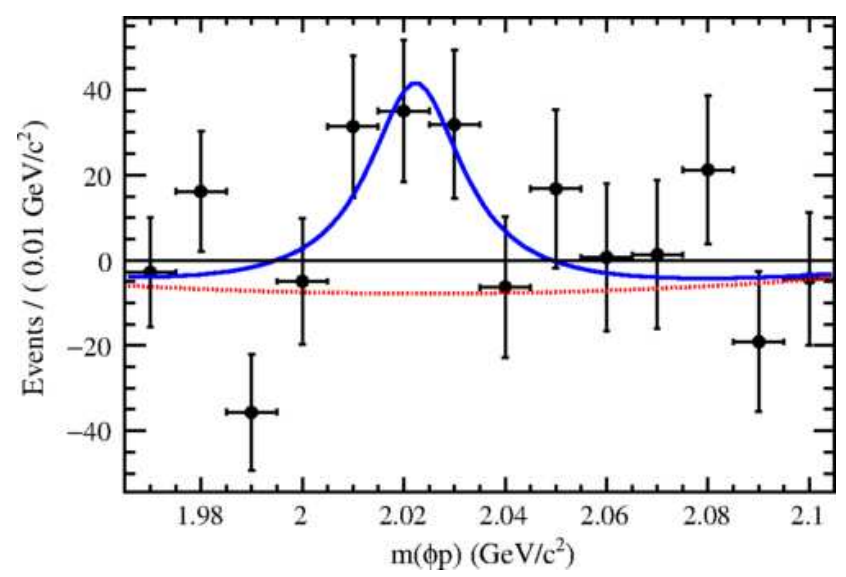

Figure 1: The background-subtracted distribution of $m(\phi p)$. The points are the data. The solid blue curve shows the total fitting results. The red dotted curve shows the fitted phase space component.

\section{Hadronic decays of $\Omega_{c}^{0}$ ground state}

The $\Omega_{c}^{0}$ charmed baryon is a combination of $c s s$ and can be interpreted as a heavy quark $(c)$ and a light diquark $(s s)$. There are four ground-state singly charmed baryons, $\Lambda_{c}^{+}, \Xi_{c}^{0}, \Xi_{c}^{+}$, and $\Omega_{c}^{0}$. All of them decay predominantly through the weak decay $c \rightarrow s W^{+}$. Only the $\Omega_{c}^{0}$ has the two same flavor light quarks among the four ground-states, and this leads to many decay diagrams producing 
the same final states. There is less experimental information on its hadronic decays than one for the other ground states $\left(\Lambda_{c}^{+}, \Xi_{c}^{0}\right.$, and $\left.\Xi_{c}^{+}\right)$.

We study the hadronic decays of the $\Omega_{c}^{0}$, and perform the precise measurements of the relative branching fractions of the $\Omega_{c}^{0}$ into $\Omega^{-} \pi^{+} \pi^{0}, \Omega^{-} \pi^{+} \pi^{-} \pi^{+}, \Xi^{-} K^{-} \pi^{+} \pi^{+}$, and $\Xi^{0} K^{-} \pi^{+}$, as well as the first measurements of the relative branching fractions of the $\Omega_{c}^{0}$ into $\Xi^{-} \bar{K}^{0} \pi^{+}, \Xi^{0} \bar{K}^{0}$, and $\Lambda \bar{K}^{0} \bar{K}^{0}$, all with respect to the $\Omega^{-} \pi^{+}$[3]. In addition, we measure an upper limit of the relative branching fraction of the $\Omega_{c}^{0}$ into $\Sigma^{+} K^{-} K^{-} \pi^{+}$. The resonant substructures are observed in the decays, $\rho^{+}$ in $\pi^{+} \pi^{0}$ via $\Omega_{c}^{0} \rightarrow \Omega^{-} \pi^{+} \pi^{0}, \Xi(1530)^{0}$ in $\Xi^{-} \pi^{+}$via $\Omega_{c}^{0} \rightarrow \Xi^{-} \pi^{+} K^{-} \pi^{+}$, and $K^{*}$ in $K^{-} \pi^{+}$via $\Omega_{c}^{0} \rightarrow$ $\Xi^{-} \pi^{+} K^{-} \pi^{+}$, and $\Omega_{c}^{0} \rightarrow \Xi^{0} \pi^{+} K^{-}$. These results will spur further theoretical work to understand the internal structure charmed baryons.
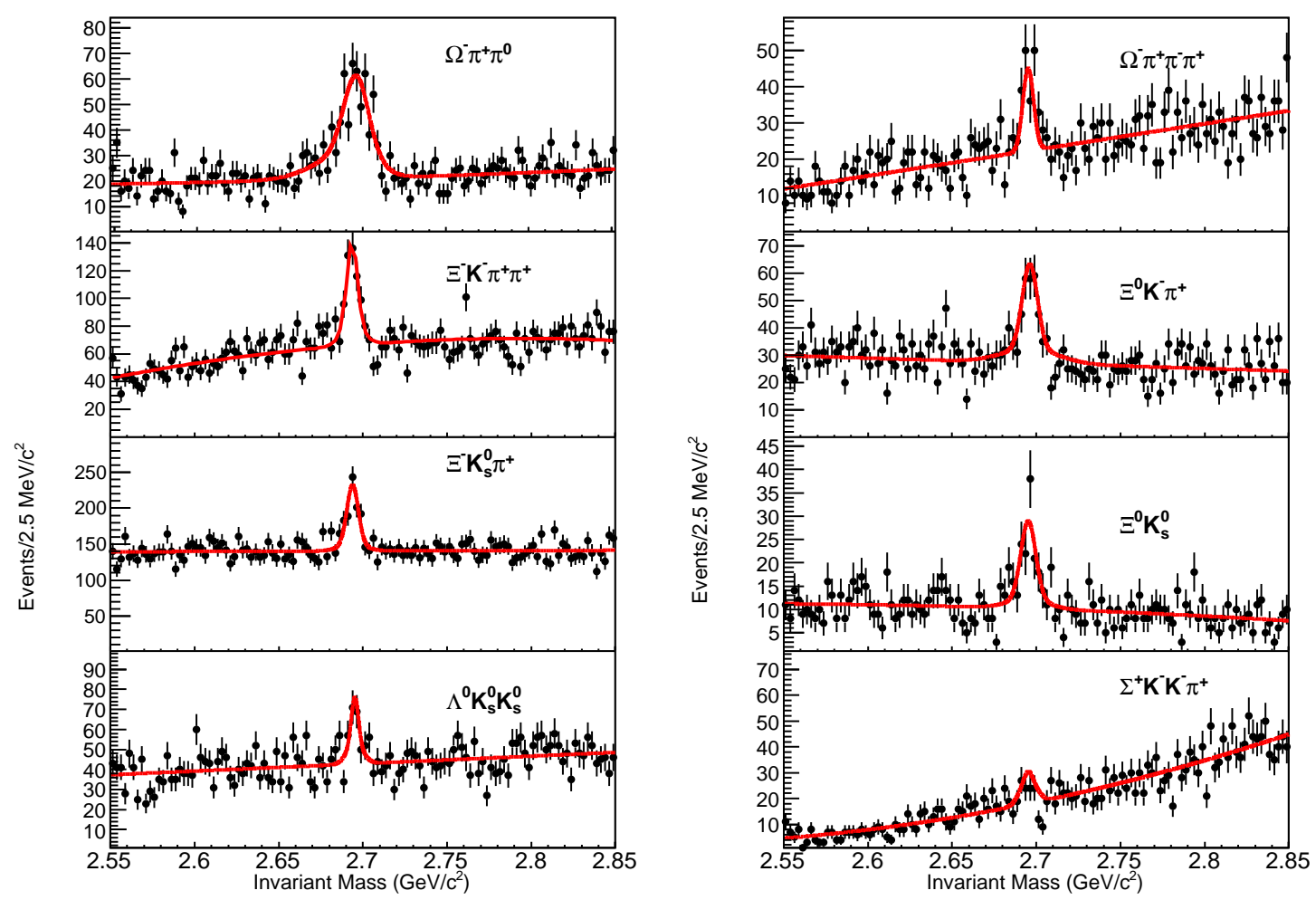

Figure 2: Invariant mass distributions. The black points are the data. The red solid curves are the fit results.

\section{Observation of excited states $\Omega_{c}^{* 0}$}

We confirm four narrow excited states of the $\Omega_{c}(3000), \Omega_{c}(3050), \Omega_{c}(3066), \Omega_{c}(3090)$ in the $\Xi_{c}^{+} K^{-}$mass spectrum [4]. These excited states have been observed togther with the other state $\Omega_{c}(3119)$ by the LHCb Collaboration [5]. The masses from the Belle are consistent with the results from the $\mathrm{LHCb}$. The five states, each with one unit of orbital angular momentum between the charm quark and the light duquark, are naturally predicted by the heavy-quark-light-diquark model [6] The different production between the Belle and LHCb may shed light on the nature of these states. 


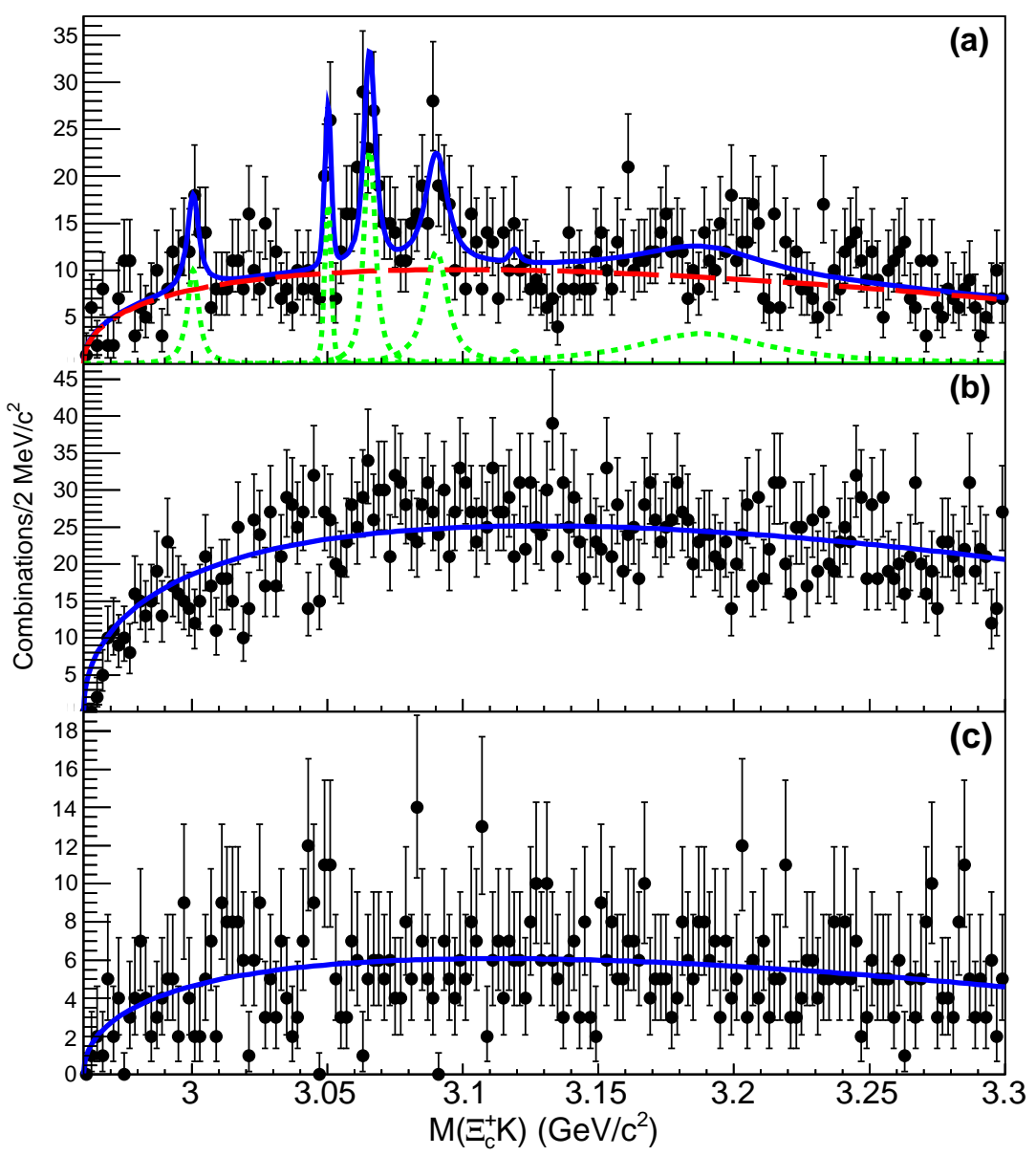

Figure 3: (a)The $\Xi_{c}^{+} K^{-}$mass spectrum. The solid blue curves are the fit. The red long-dashed curve is for the background, and the green dashed curve is six Voigtian functions. (b)the $\Xi_{c}^{+} K^{+}$(wrong sign) mass distribution with the background fit. (c)The mass distribution for the sidebands to the $\Xi_{c}^{+}$candidates in combination with $K^{-}$candidates

\section{Observation of $\Xi_{c}(2930)^{0}$}

The $\Xi_{c}(2930)^{0}$ has been taken into account in many theoretical models despite a one-star rating for this state in PDG [7]. We report the first observation of the $\Xi_{c}(2930)^{0}$ in its decay to $K^{-} \Lambda_{c}^{+}$ via $B^{-} \rightarrow K^{-} \Lambda_{c}^{+} \bar{\Lambda}_{c}^{-}$with a significance greater than $5 \sigma$ [8]. The measured mass and width are $2928.9 \pm 3.0$ (stat.) ${ }_{-12.0}^{+0.9}$ (syst.) $\mathrm{MeV} / \mathrm{c}^{2}$ and $19.5 \pm 8.4$ (stat.) ${ }_{-7.8}^{+5.9}$ (syst.), respectively. These results are consistent with the results by the BaBar Collaboration [9]. The product branching fraction is measured to be $\mathcal{B}\left(B^{-} \rightarrow \Xi_{c}(2930)^{0}{\overline{\Lambda_{c}^{-}}}^{-}\right) \mathcal{B}\left(\Xi_{c}(2930)^{0} \rightarrow K^{-} \Lambda_{c}^{+}\right)=1.73 \pm 0.45$ (stat.) \pm 0.21 (syst.) $\times 10^{-4}$. We also measure $\mathcal{B}\left(B^{-} \rightarrow K^{-} \Lambda_{c}^{+} \bar{\Lambda}_{c}^{+}\right)=4.80 \pm 0.43$ (stat.) \pm 0.60 (syst.) $\times 10^{-4}$ with improved precision. The assignment of this state in the theoretical models requires the measurement of the spin parity which will be possible with Belle II detector. 


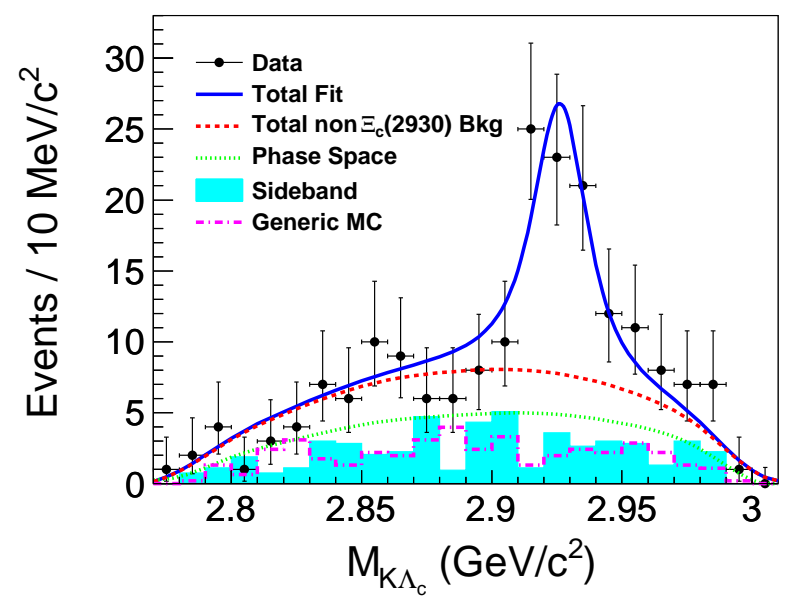

Figure 4: The $K^{-} \Lambda_{c}^{+}$mass spectrum. The details are shown in this plot.

\section{Production rates of baryons}

The study of the production cross sections of various baryons or mesons is useful to understand the intarnal stucture of hadrons, and extruct exotic states which may show different behavior on the mass dependnece of cross sections. We measure the inclusive production cross sections of hyperons and charmed baryons[10].
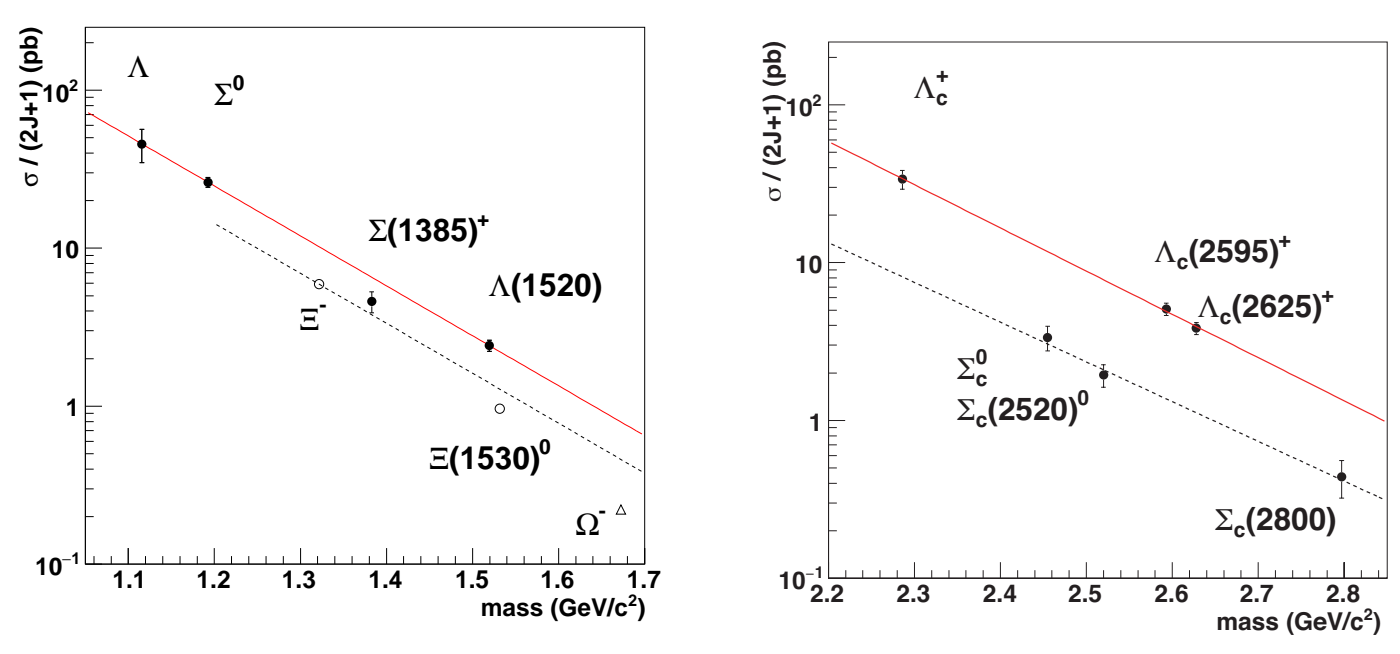

Figure 5: Scaled direct production cross section as a function of mass of hyperons(left plot) and charmed baryons(right plot). The black points are the data. The solid and dashed lines show the fit results using exponential functions.

The feed-down contributions from heavy particles are subtracted by using this data, and the direct production cross sections are measured for the first time. The production cross sections 
divided by the number of spin states for hyperons follow an exponential function with a single slope parameter except for the $\Sigma(1385)^{+}$resonance. Suppressionfor $\Sigma(1385)^{+}$and $\Xi(1530)^{0}$ hyperons is observed. A factor of three difference for $\Lambda_{c}^{+}$states over $\Sigma_{c}$ states is observed. This observation suggests a diquark structure for these baryons.

\section{References}

[1] R. Aaij et al. (LHCb Collaboration), Phys. Rev. Lett. 115 (2015) 072001.

[2] B. Pal et al. (Belle Collaboration), Phys. Rev. D 96 (2017) 051102(R).

[3] J. Yelton et al. (Belle Collaboration), Phys. Rev. D 97 (2018) 032001.

[4] J. Yelton et al. (Belle Collaboration), Phys. Rev. D 97 (2018) 051102.

[5] R. Aaij et al. (LHCb Collaboration), Phys. Rev. Lett. 118 (2017) 182001.

[6] D. Lichtenberg, Nuovo Cimento Soc. Ital.Fis. 28A (1975) 563.

[7] M. Tanabashi et al. (Particle Data Group), Phys. Rev. D 98 (2018) 030001.

[8] Y. B. Li et al. (Belle Collaboration), Eur. Phys. J. 78 (2018) 252.

[9] B. Aubert et al. (BaBar Collaboration), Phys. Rev. D 77 (2008) 031101.

[10] M. Niiyama et al. (Belle Collaboration), Phys. Rev. D 97 (2018) 072005. 